\title{
Structure Constant of the Yang-Lee Edge Singularity
}

\author{
TOMASZ WYDRO \\ Laboratoire de Physique des Materiaux \\ UMR CNRS 7556, Universite Henri Poincare \\ Nancy 1, B.P. 239, F-54506 Vandoeuvre-les-Nancy Cedex, France \\ wydro@lpm.u-nancy.fr \\ JOHN F. McCABE \\ 412 Morris Ave., \# 34, Summit, NJ 07901, USA \\ jfmccabe2@earthlink.net
}

November 20, 2018

\begin{abstract}
This paper studies the Yang-Lee singularity of the 2-dimensional Ising model on the cylinder via transfer matrix and finite-size scaling techniques. These techniques enable a measurement of the 2-point and 3-point correlations and a comparison of a measurement of a corresponding universal amplitude with a prediction for the amplitude from the $\left(A_{4}, A_{1}\right)$ minimal conformal field theory.
\end{abstract}

PACS codes: 05.50.+q, 05.70.Jk, 11.25.Hf.

Keywords: Ising model, Yang-Lee singularity, Conformal Field Theory.

In 1985, Cardy [1] provided evidence that the Yang-Lee edge singularity [2] of the 2-dimensional (2D) Ising model could be described by the non-unitary $\left(A_{4}, A_{1}\right)$ minimal conformal field theory (CFT) $[3,4]$ of the ADE classification [5]. Cardy's identification provided several CFT predictions for the Yang-Lee edge singularity of the 2D Ising model. The predictions for the central charge, the exponent $\nu$, and the low energy excitation spectrum have been confirmed by numerical measurements on lattice spin models [6].

Cardy also determined the forms of 2-point and 3-point correlations of the single primary field in the $\left(A_{4}, A_{1}\right)$ minimal CFT [1]. These correlations define a universal amplitude, which is known as a structure constant $[3,7]$. The predictions of universal amplitudes are an important advance that CFT brought to the understanding of critical points. Since these predictions of CFT are based on nontrivial applications of crossing symmetries, they deserve to be experimentally tested [3]. 
While lattice models have been used to numerically test some predictions of universal amplitudes in unitary minimal CFTs $[3,7,8]$, no tests of such predictions have been done for the non-unitary CFTs. This article tests such a prediction for a non-unitary CFT by numerically measuring spin correlations at the Yang-Lee edge singularity of the 2D Ising model. The measurements enable a test of the CFT prediction of a universal amplitude in the non-unitary $\left(A_{4}, A_{1}\right)$ minimal CFT.

As pointed out by Cardy [1], the non-unitary $\left(A_{4}, A_{1}\right)$ minimal CFT has a single primary field $\phi(z, \bar{z})$, which has left and right conformal weights of $-1 / 5$ and a scaling dimension $x$ of $-2 / 5$. The 2 -point and 3 -point correlations of the primary field $\phi(z, \bar{z})$ have the respective forms:

$$
G_{\phi \phi}\left(z_{1}, \overline{z_{1}}, z_{2}, \overline{z_{2}}\right)=\mid\left(z_{1}-\left.z_{2}\right|^{4 / 5}\right.
$$

and

$$
G_{\phi \phi \phi}\left(z_{1}, \overline{z_{1}}, z_{2}, \overline{z_{2}}, z_{3}, \overline{z_{3}}\right)=C\left|\left(z_{1}-z_{2}\right)\left(z_{2}-z_{3}\right)\left(z_{3}-z_{1}\right)\right|^{2 / 5} .
$$

With the Coulomb gas formalism [7], Cardy showed that the single structure constant, $C$, of the non-unitary $\left(A_{4}, A_{1}\right)$ minimal CFT has the value [1]:

$$
C=\sqrt{-\frac{[\Gamma(6 / 5)]^{2} \Gamma(1 / 5) \Gamma(2 / 5)}{\Gamma(3 / 5)[\Gamma(4 / 5)]^{3}}}
$$

In this article, numerical measurements at the Yang-Lee edge singularity check this CFT prediction.

The numerical measurements were made for the $2 \mathrm{D}$ ferromagnetic Ising model whose Hamiltonian, $H$, is:

$$
H=-\sum_{j=1}^{M} \sum_{i=1}^{N}\left[J\left(S_{i, j} S_{i, j+1}+S_{i, j} S_{i+1, j}\right)+h S_{i, j}\right] .
$$

In the ferromagnetic Hamiltonian, the spin-spin coupling $J$ is positive. For the Ising model, the Yang-Lee edge singularity occurs at temperatures above the critical temperature and for purely imaginary values of the magnetic field, $h$, i.e., $h=i B$ with $B$ real [2]. In particular, spin correlations were measured at a temperature, $\mathrm{T}$, for which $J / k_{B} T=0.1$

For the above Hamiltonian, the transfer matrix was used to evaluate the correlations. In particular, 2-spin and 3-spin correlations were evaluated on torii of length, $M$, and of various diameters, $N$. In these evaluations, $M$ was always much larger than $N$, i.e., $\mathrm{M}=512$ and $\mathrm{N}=3-8$. Thus, the measured correlations had the same distance behavior as correlations on an infinitely long cylinder when distances between spin fields were very small compared to $M$.

From the numerical evaluations, finite-size scaling provided the tool for extracting values of physical properties in the thermodynamic limit [9]. In particular, the spin correlations are measured at special values of the purely imaginary magnetic field, $h(N)=i B_{Y L}(N)$. Each special value , $B_{Y L}(N)$, satisfies the phenomenological renormalization group (PRG) equation for infinite cylinders of diameters $(N-1)$ and $N$ :

$$
\frac{\xi\left(i B_{Y L}(N), N-1\right)}{N-1}=\frac{\xi\left(i B_{Y L}(N), N\right)}{N} .
$$


In the PRG equation, $\xi(i B, N)$ is the spin-spin correlation length on the infinite cylinder of diameter $N$ when the magnetic field is $i B$. The PRG equation imposes that the spin-spin correlation length scales linearly with $N$ as $N \rightarrow \infty$. When evaluated at PRG values of the magnetic field, other physical quantities will scale with the width, $N$, to their values in the thermodynamic limit, i.e., near the Yang-Lee edge singularity [10, 6].

On an infinite cylinder of width $N$, CFT predicts that correlations will depend exponentially on the distances between the fields in the correlations when said distances are large compared the cylinder's diameter, $N$ [11]. For a 2-point correlation, the correlation length is related to the scaling dimension, $x$, of the two conformal fields of the correlation. In particular, the 2-point correlation has the form $\exp \left(-2 \pi x\left(y_{1}-y_{2}\right) / N\right)$ when $\left|y_{1}-y_{2}\right|>>N$. Here, $y_{1}$ and $y_{2}$ are the positions of the two fields of the correlation along the axis of the infinite cylinder. For the 3-point correlation, the exponential behavior on the distances between the fields of the correlation is again fixed by the scaling dimensions, $x^{\prime} s$, of the various fields of the correlation.

At the Yang-Lee edge singularity of the 2D Ising model, we used the amplitudes of the 2-spin and 3-spin correlations, i.e., $A_{s s}$ and $A_{s s s}$, respectively, to evaluate the 3 -spin structure constant. In particular, the measured values of the 3-spin structure constant, $C(N)$, were obtained from the relation:

$$
C(N)=\frac{A_{s s s}\left(i B_{Y L}(N)\right)}{\left[A_{s s}\left(i B_{Y L}(N)\right]^{3 / 2}\right.} .
$$

Here, $A_{s s}\left(i B_{Y L}(N)\right)$ and $A_{s s s}\left(i B_{Y L}(N)\right)$, are the measured amplitudes of the 2 -spin and 3 -spin correlations, respectively, at PRG values of the magnetic field. Similarly, we used the PRG measurements of the correlation length $\xi(N)$ to measure the conformal dimension, $x$, of the spin field, i.e., $x(N)=$ $N /\left[2 \pi \xi\left(i B_{Y L}(N)\right)\right]$. The scaling behavior of these physical quantities with the cylinder's width, $N$, was used to obtain their values in thermodynamic limit, i.e., as $N \rightarrow \infty$.

Table 1 summarizes our transfer matrix results ${ }^{1}$ for $M=512$ and $J / k_{B} T=$ 0.1 .

\begin{tabular}{clll}
\hline$N$ & $B_{Y L}(N)$ & $x(N)$ & $|C(N)|$ \\
\hline 3 & 0.184802 & 0.353929 & 1.80838 \\
4 & 0.183348 & 0.376870 & 1.83711 \\
5 & 0.183064 & 0.385748 & 1.85736 \\
6 & 0.182982 & 0.390108 & 1.87054 \\
7 & 0.182951 & 0.392693 & 1.87937 \\
8 & 0.182946 & 0.392911 & 1.88633 \\
$\ldots$ & $\ldots$ & $\ldots$ & $\ldots$ \\
$\infty$ & $\ldots$ & $0.398(2)$ & $1.923(13)$ \\
$C F T$ & - & 0.4 & 1.9113 \\
\hline
\end{tabular}

Table 1: PRG Measurements of conformal dimension and structure constant.

In these measurements of correlations, adjacent spin fields were separated

${ }^{1}$ While the $B_{Y L}(N)$ 's for $\mathrm{N}=3$ - 7 were evaluated from the PRG equation, $B_{Y L}(N)$ for $\mathrm{N}=8$ was only approximately evaluated for numerical reasons. $B_{Y L}(8)$ was determined from the $B_{Y L}(N)$ 's for $\mathrm{N}=3-7$ by assuming a leading finite-size scaling behavior. 
by distances that were large compared to $N$ and small compared to $M$. In particular, we measured correlations in which the distances between adjacent spin fields were between about $4 N$ and $0.06 M$. For such distances, the CFT prediction of an exponential dependence on the distances between fields was obtained. In Table 1 , the $\infty$ line shows values obtained by extrapolating our measurements to the thermodynamic limit, i.e., $N=\infty$. The extrapolated values were obtained from nonlinear fits of the measured values of $x(N)$ and $|C(N)|$ to functions of form $f(N)=f(\infty)+f_{1} N^{-\alpha}$, i.e., to account for leading finite-size corrections. In each such extrapolation, the coefficient, $f_{1}$, and the scaling power $\alpha$ were determined by finding the best fit to the measured data points. The best fits were found when the scaling corrections or $x(N)$ and $|C(N)|$ had exponents, $\alpha$, of about 2.4 and 1.2. In Table 1 , the last line shows Cardy's CFT predictions for $x$ and $|C|$ from the $\left(A_{4}, A_{1}\right)$ non-unitary minimal CFT as the model.

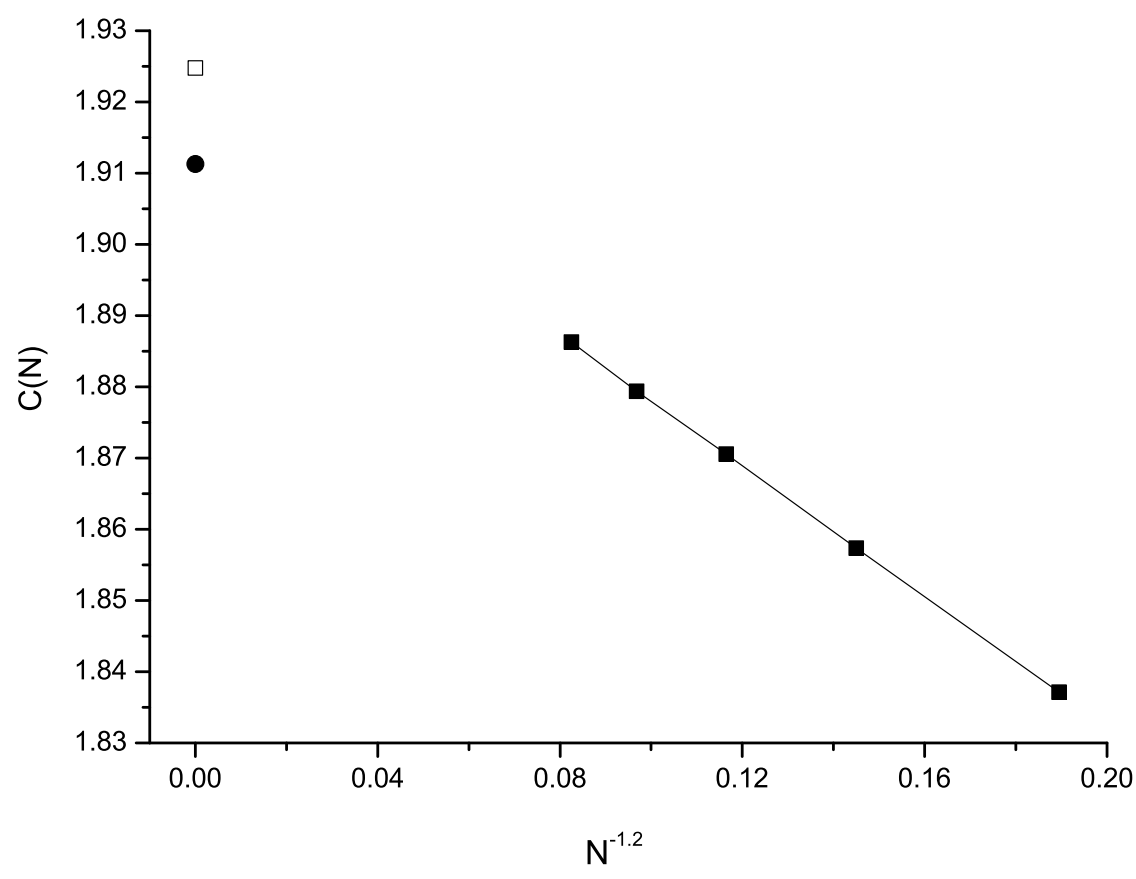

Figure 1: The measured structure constant (squares) for cylinders of diameter $N$ plotted against nonlinear fit for $N=4-8$.

Figure 1 plots our data for $|C(N)|$ and our best fit (line) based on a leading correction in $N^{-1.2}$. In Figure 1, the black squares represent the measured $C(N)$ 's, the empty square represents the value of $C(\infty)$ obtained from the best fit to the measurements, and the black circle represents Cardy's CFT prediction for $C(\infty)$.

The measured structure constants for cylinders of diameter $3-8$ exhibits an approximate finite-size scaling correction of the form $N^{-1.2}$. The finite-size 
scaling motivated fit matches the measured values of structure constant well except for the thinnest cylinder, $N=3$. There, one is not surprised that a large $N$ expansion does not work well. Finally, the dot shows the structure constant predicted by CFT.

In conclusion, our finite-size scaling measurements for the 2D Ising model at the Yang-Lee edge singularity produce a value of the structure constant that agrees well with that of the $\left(A_{4}, A_{1}\right)$ minimal CFT. These measurement confirm the CFT prediction of this universal amplitude at the Yang-Lee edge singularity of the 2D Ising model.

\section{References}

[1] J.L. Cardy, Phys. Rev. Lett. 54, 1354 (1985).

[2] C.N. Yang and T.D. Lee, Phys. Rev. 87, 404 and 410 (1952).

[3] A.A. Belavin, A.M. Polyakov, and A.B. Zamolodchikov, Nucl. Phys. B241, 333 (1984).

[4] D. Friedan, Z. Qiu, and S. Shenker, Phys. Rev. Lett. 52, 1575 (1984), and Comm. Math. Phys. 107, 535 (1986).

[5] A. Cappelli, C. Itzykson, and J.-B. Zuber, Nucl. Phys. B280, 445 (1987), and Comm. Math. Phys. 113, 1 (1987); A. Kato, Mod. Phys. Lett. A2, 585 (1987); for a review see C. Itzykson and J.-M. Drouffe, Statistical Field Theory (Cambridge University Press, U.K., 1989) Ch. IX.

[6] C. Itzykson, H. Saleur, and J.-B. Zuber, Europhys. Lett. 2, 91 (1986); K. Uzelac and R. Jullien, J. Phys. A14, L151 (1981); J.F. McCabe and T. Wydro, Int. J. of Mod. Phys. B20, 495 (2006).

[7] Vl.S. Dotsenko and V.A. Fateev, Nucl. Phys. B240, 312 (1984), and B251, 691 (1985).

[8] G.T. Barkema and J. McCabe, J. Stat. Phys. 84, 1067 (1996); J. McCabe and T. Wydro, Int. J. of Mod. Phys. A13, 1013 (1998); G. von Gehlen, V. Rittenberg, and T. Vescan, J. Phys. A20, 2577 (1987).

[9] M.E. Fisher and M.N. Barber, Phys. Rev. Lett. 28, 1516 (1972); for a review see M. Henkel, Conformal Invariance and Critical Phenomena (Springer-Verlag, Germany, 1999) Ch. 3.

[10] M.P. Nightingale, Physica A83, 561 (1976); and in Finite Size Scaling and Numerical Simulation of Statistical Systems, edited by Ed. V. Privman (World Scientific, Singapore, 1990) Ch. VII.

[11] J.L. Cardy, J. Phys. A17, L385 (1984), and Nucl. Phys. B270, 186 (1986); for a review see M. Henkel, Conformal Invariance and Critical Phenomena (Springer-Verlag, Germany, 1999) Ch. 13. 